\title{
Dynamic Analysis of a Micro Drilling System by the Modified Transfer Matrix Method
}

\author{
An-Chen Lee ${ }^{1, \mathrm{a}}$ and Danh-Tuyen Nguyen ${ }^{2}$ \\ ${ }^{1,2}$ Department of Mechanical Engineering, National Chiao Tung University, 1001 Ta Hsueh Road, Hsinchu 30010, Taiwan
}

\begin{abstract}
Normally, breakage is the main cause of failure of a micro drilling system, of which coupling effect of lateral and torsional displacements plays the most important role. This research developed a modified transfer matrix method to investigate the dynamics of a micro drilling system focusing on displacements induced by not only lateral, shear, torsional deformations, but also the coupling effect between lateral and torsional vibrations. The micro drilling system is modeled as pre-twisted rotating Timoshenko beam elements with a continuous-system concept. Each element includes components of linear bearings, axial drilling force, gyroscopic moments, and eccentricity. The overall transfer matrix of the system is developed to determine the state vector of the system including displacements and forces. Finally, a numerical example was presented, where the lateral and torsional displacements and critical speed of the micro drilling system at the micro drill's tip were presented.
\end{abstract}

\section{Introduction}

Nowadays, with the development of science and technology, there is an increasing demand for producing micro holes in some devices, for example, cameras, mobile phones, computers, medical instruments, micro dies and molds, fuel injection nozzles, watches, bearings, especially printed circuit boards (PCB). In methods to manufacture micro holes now, such as using micro mechanical machining or micro drilling, electron beam machining, electrical discharge machining, and laser machining, the micro drilling has higher accuracy, greater productivity, and more economical efficiency, compared with other approaches [1-3]. Hence, there are more and more researchers making great efforts to improve the ability of micro drilling to meet that demand. To achieve that purpose, it is necessary to understand the dynamic characteristics of micro drilling process $[4,5]$. The transverse displacement affects hole's accuracy, and exhibits the maximum displacements if its operating speed is equal to a critical one. In the past, several researches on dynamics of micro drilling were published. To name few, Yang studied transverse displacement of a micro drill tip, but a lot of dynamic properties of the micro drilling system on the drilling process are not considered, such as eccentricity, gyroscopic moments, etc. [6]. Ehmann modeled micro drills as long twisted beams including effects of transverse shear, rotary inertia and gyroscopic moments during drilling. Models for the critical speeds and critical bucking loads were developed by the finite element method. However, the beams did not include the effect of eccentricity of the drilling system and the torsional displacements were ignored [7]. Zhaojun modeled a micro-drill-spindle system as
Timoshenko beam elements. Besides considerations of transverse shear, rotary inertia and gyroscopic moments similar to Ehmann's models, the element models also included the effects of eccentricity of the spindle-clampdrill system, axial drilling force, and bearings. Nevertheless, the element's torsional displacement was also ignored [8]. Ozdoganlar modeled the flute part of a micro drill, and other parts as a beam including torsional displacement and one-dimensional beams, respectively. However, the models did not consider the effect of eccentricity of the drilling system $[9,10]$.

In this research, the underlying micro drilling system includes a micro drill grasped in a spindle, which is supported by identical linear bearings. It is modeled as pre-twisted rotating Timoshenko beam elements with a continuous-system concept. Each element includes effects of linear bearings, axial drilling force, gyroscopic moments, and eccentricity. Its orientation is described by Euler angles. Then the kinetic, potential energies, and work done by non-conservative forces are developed, and the Hamilton's principle is applied to obtain its motion equations, as well as natural boundary conditions with respect to the fixed coordinates. The harmonic balance method is used to solve those equations. The state vector of the system containing displacements and forces is developed by the transfer matrix method (TMM). The most significant advantage of this method is that it does not require the storage and manipulation of large system arrays. Therefore, there is less demand on computer memory. After developing local transfer matrices of system elements and bearings, the overall transfer matrix of the system is developed to determine the state vector. Finally, a numerical example was presented and the results of the dynamic analysis showed the effects of

\footnotetext{
${ }^{\mathrm{a}}$ Corresponding author: aclee@mail.nctu.edu.tw
} 
external forces, bearing coefficients, and eccentricity to the displacements and critical speeds of the micro drilling system at the micro drill tip.

\section{Motion Equations of the Drilling System Element}

The structure of the micro drilling system can be simplified as shown in Fig.1. The micro drill $\mathrm{E}$ is grasped in the clamp C-D that is connected to the spindle A by a small shaft B. The system is supported by two identical linear bearings, and applied by an axial force $F_{a}$, and a torque $T$ during drilling the work-piece W. The whole system's dimensions and parameters are shown in table 1 $[8,11]$.

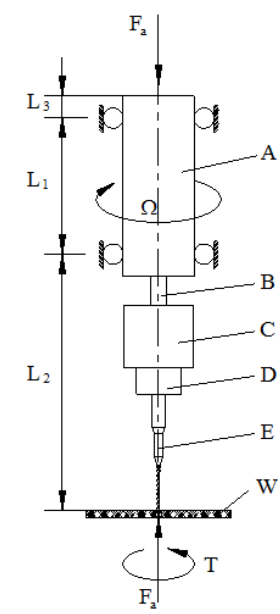

(a) Structure of micro drilling system

Figure 1. Micro drilling system

Because the axial displacement is much smaller than the other displacements, it is neglected in this research [12-14]. The micro drill and spindle of micro drilling system are modeled pre-twisted rotating Timoshenko beam elements as shown in Fig. 2.

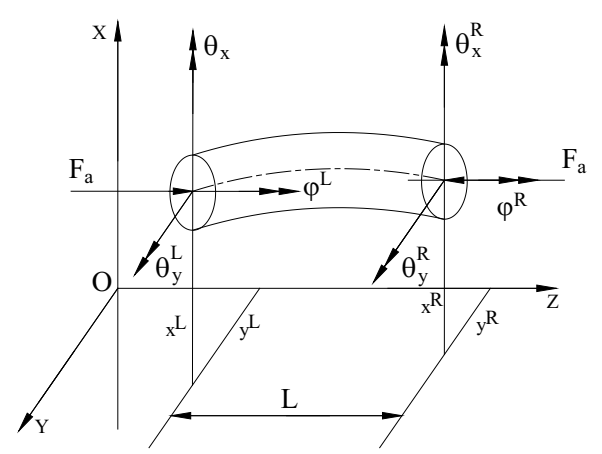

Figure 2. Pre-twisted rotating Timoshenko beam element

The motion equations of the pre-twisted rotating Timoshenko beam element are developed by applying the extended Hamilton's principle. According to this principle, for a rotating element with kinetic energy $E_{k}$, potential energy $E_{p}$, virtual work $W$ done by the nonconservative force, its governing equation motions can be obtained by the following equation.

$$
\delta \int_{t_{1}}^{t_{2}}\left(E_{k}-E_{p}+W\right) d t=0
$$

The kinetic energy $E_{k}$ includes translational and rotational ones. To make it simple for developing the rotational kinetic energy, the Euler angles $(\phi, \theta, \psi)$ as shown in Fig. 3(a) are used. They were introduced by Leonhard Euler to describe orientations of rotating elements and explained via the following steps: (1) rotate the $X Y Z$ initial coordinate system, parallel to the fixed coordinate system, about the $Z$ axis by an angle $\phi$ to lead to the $X^{\prime} Y^{\prime} Z^{\prime}$ coordinate system, (2) rotate $X^{\prime} Y^{\prime} Z^{\prime}$ about the $X^{\prime}$ axis by an angle $\theta$ to lead to the $U^{\prime} V^{\prime} W^{\prime}$ coordinate system, (3) rotate $U^{\prime} V^{\prime} W^{\prime}$ about the $W^{\prime}$ axis by an angle $\psi$ to produce the $U V W$ principal coordinate system. As the rotating element is deflected in position and orientation, the angular displacements as shown in Fig. 3(b) are the projections of angle $\theta$, measuring counterclockwise from the $Z$ fixed axis to the $W$ spin axis, onto $Y Z$ and $X Z$ planes, thus

$$
\left\{\begin{array}{l}
\theta_{x}=\theta \cos \phi \\
\theta_{y}=\theta \sin \phi
\end{array}\right.
$$

Furthermore, the spin angle about the $W$ axis is obtained as $\Phi=\phi+\psi$ from the geometric configuration of a rotating element with a very small oblique angle $\theta$. Applying the coordinate transformation formula, the components of the angular velocities in directions of the principal coordinates are obtained $[11,15]$.

$$
\left\{\begin{array}{l}
\omega_{u}=\dot{\theta} \cos \psi+\dot{\phi} \sin \theta \sin \psi \\
\omega_{v}=-\dot{\theta} \sin \psi+\dot{\phi} \sin \theta \cos \psi \\
\omega_{w}=\dot{\psi}+\dot{\phi} \cos \theta
\end{array}\right.
$$

Take the mass center $C$ of the cross-section of the rotating element as the origin of the $U V W$ principal coordinate system. Its kinetic energy $E_{k}$ is calculated in the fixed coordinate system as follows:

$$
E_{k}=\frac{1}{2} \rho \int_{0}^{L}\left[A\left(\dot{x}_{c}^{2}+\dot{y}_{c}^{2}\right) \frac{1}{2}\left(I_{u} \omega_{u}^{2}+I_{v} \omega_{v}^{2}+I_{p} \omega_{w}^{2}\right)\right] d Z
$$

where $A$ is the cross sectional area, $\rho$ is mass density. $I_{u}$, and $I_{v}$ are area principal moments of inertia, $I_{p}$ is area polar moment of inertia. The $\omega_{u}, \omega_{v}, \omega_{w}$ are angular velocities about principal axes $U, V, W$, respectively. 


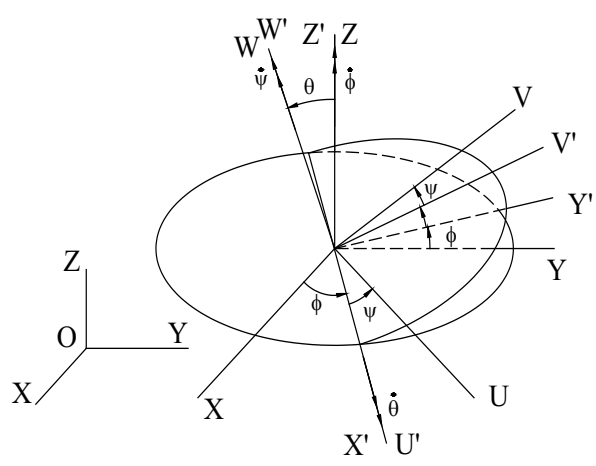

(a) Euler angles of a rotating element

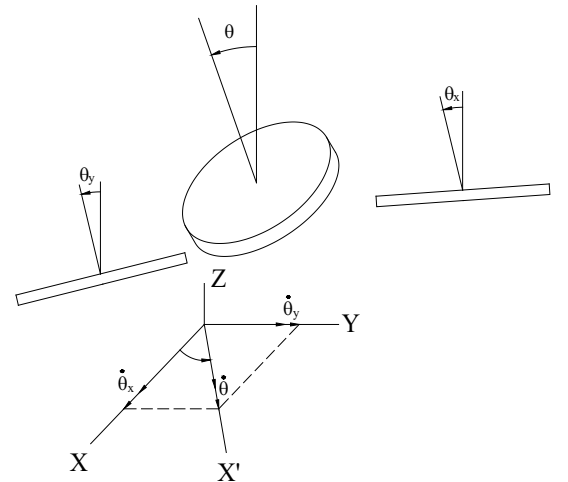

(b) Element projection

Figure 3. Orientation of a rotating element

Table 1. Structure dimensions of the micro drilling system

\begin{tabular}{|c|c|c|c|c|c|}
\hline \multicolumn{2}{|l|}{ Name of shafts } & Shaft symbol & $\begin{array}{l}\text { Diameter } \\
(\mathrm{mm})\end{array}$ & $\begin{array}{l}\text { Length } \\
(\mathrm{mm})\end{array}$ & $\begin{array}{l}\text { Number of } \\
\text { divided elements }\end{array}$ \\
\hline \multicolumn{2}{|l|}{ Spindle } & A & 22 & 60 & 3 \\
\hline \multicolumn{2}{|l|}{ Shaft } & $\mathrm{B}$ & 6.3 & 9 & 1 \\
\hline \multicolumn{2}{|l|}{ Up clamp } & $\mathrm{C}$ & 22.5 & 19 & 1 \\
\hline \multicolumn{2}{|l|}{ Down clamp } & $\mathrm{D}$ & 14 & 8 & 1 \\
\hline \multirow{5}{*}{ Micro drill } & Shank & $\mathrm{S}_{1}$ & 3 & 10 & 1 \\
\hline & Cone 1 & $\mathrm{C}_{1}$ & & 4.1 & 5 \\
\hline & Cylinder & $\mathrm{S}_{2}$ & 0.8 & 2.6 & 1 \\
\hline & Cone 2 & $\mathrm{C}_{2}$ & & 0.933 & 5 \\
\hline & Flute & $\mathrm{F}_{1}$ & 0.3 & 5 & 20 \\
\hline \multicolumn{2}{|c|}{ Positions of bearings (mm) } & $\mathrm{L}_{1}=11.25, \mathrm{~L}_{3}=37.5$ & & & \\
\hline
\end{tabular}

The Fig 4 shows the whirling orbit of a rotating element which is in asymmetric, eccentric form. From the geometric relations, the coordinates of the mass center $C$ can be calculated as follows:

$$
\begin{gathered}
{\left[\begin{array}{l}
x_{c} \\
y_{c}
\end{array}\right]=\left[\begin{array}{l}
x \\
y
\end{array}\right]+\left[\begin{array}{l}
e_{x} \\
e_{y}
\end{array}\right]} \\
{\left[\begin{array}{l}
e_{x} \\
e_{y}
\end{array}\right]=\left[\begin{array}{ll}
\cos (\Omega t+\varphi+\beta) & -\sin (\Omega t+\varphi+\beta) \\
\sin (\Omega t+\varphi+\beta) & \cos (\Omega t+\varphi+\beta)
\end{array}\right]\left[\begin{array}{l}
e_{u} \\
e_{v}
\end{array}\right]}
\end{gathered}
$$

where, $\Omega$ is rotating speed , $\varphi$ is torsional angle, $\beta$ is the initial twist angle between principle axes $U$, and $X$ axis, $t$ is time, $e_{u}, e_{v}$ are eccentricities corresponding to $U, V$ coordinates, respectively. Substituting Eq. 6 into Eq. 5, and differentiating it yields,

$$
\begin{gathered}
\dot{x}_{c}=\dot{x}-e_{v}(\Omega+\dot{\varphi}) \cos (\Omega t+\varphi+\beta) \\
-e_{u}(\Omega+\dot{\varphi}) \sin (\Omega t+\varphi+\beta) \\
\dot{y}_{c}=\dot{y}+e_{u}(\Omega+\dot{\varphi}) \cos (\Omega t+\varphi+\beta) \\
-e_{v}(\Omega+\dot{\varphi}) \sin (\Omega t+\varphi+\beta)
\end{gathered}
$$

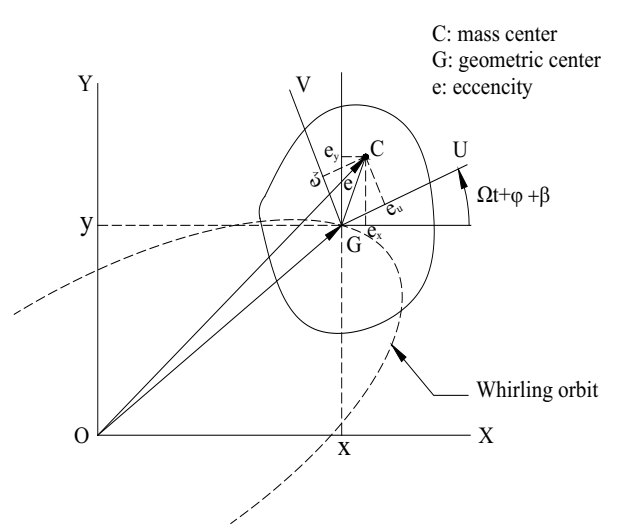

Figure 4. Whirling orbit of a rotating element 
Replacing Eqs. 2, 3, 7, and 8, and $\Phi=\Omega t+\varphi+\beta$ into Eq. 4, one gets

$$
\begin{aligned}
E_{k} & =\frac{1}{2} \rho \int_{0}^{L}\left\{A \left[\dot{x}^{2}-2 \dot{x} e_{v}(\Omega+\dot{\varphi}) \cos (\Omega t+\varphi+\beta)\right.\right. \\
& -2 \dot{x} e_{u}(\Omega+\dot{\varphi}) \sin (\Omega t+\varphi+\beta) \\
& +\dot{y}^{2}+2 \dot{y} e_{u}(\Omega+\dot{\varphi}) \cos (\Omega t+\varphi+\beta) \\
& -2 \dot{y} e_{v}(\Omega+\dot{\varphi}) \sin (\Omega t+\varphi+\beta) \\
& \left.+(\Omega+\dot{\varphi})^{2} e^{2}\right]+I_{p}(\Omega+\dot{\varphi})^{2} \\
& +I_{p}(\Omega+\dot{\varphi})\left(\dot{\theta}_{x} \theta_{y}-\dot{\theta}_{y} \theta_{x}\right)+I\left(\dot{\theta}_{x}^{2}+\dot{\theta}_{y}^{2}\right) \\
& +2 \Delta \dot{\theta}_{x} \dot{\theta}_{y} \sin 2(\Omega t+\varphi+\beta) \\
& \left.+\Delta\left(\dot{\theta}_{x}^{2}-\dot{\theta}_{y}^{2}\right) \cos 2(\Omega t+\varphi+\beta)\right\} d Z
\end{aligned}
$$

where, $I=\frac{1}{2}\left(I_{u}+I_{v}\right), \Delta=\frac{1}{2}\left(I_{u}-I_{v}\right)$

Assuming the rotating element as shown in Fig 5 is acted by an axial force $F_{a}$, its potential energy $E_{p}$. According to the bending and shear deformations can be described as in the following:

$$
\begin{aligned}
E_{p}=\frac{1}{2} \int_{0}^{L}\left[E(I+\Delta \cos 2(\Omega t+\varphi+\beta))\left(\theta_{x}^{\prime}\right)^{2}\right. \\
+E(I-\Delta \cos 2(\Omega t+\varphi+\beta))\left(\theta_{y}^{\prime}\right)^{2} \\
+2 \mathrm{E} \Delta \theta_{x}^{\prime} \theta_{y}^{\prime} \sin 2(\Omega t+\varphi+\beta) \\
+k_{s} G A\left(\left(x^{\prime}-\theta_{y}\right)^{2}+\left(y^{\prime}+\theta_{x}\right)^{2}\right) \\
\left.+G I_{p}\left(\varphi^{\prime}\right)^{2}-F_{a}\left(\left(x^{\prime}\right)^{2}+\left(y^{\prime}\right)^{2}\right)\right] d Z
\end{aligned}
$$

From Fig. 5, the work done by external force is

$$
\begin{aligned}
W= & V_{x}^{R} x+M_{y}^{R} \theta_{y}+V_{y}^{R} y+M_{x}^{R} \theta_{x}+T^{R} \varphi \\
& -\left(V_{x}^{L} x+M_{y}^{L} \theta_{y}+V_{y}^{L} y+M_{x}^{L} \theta_{x}+T^{L} \varphi\right)
\end{aligned}
$$

Substituting Eqs. 9, 10, and 11 into Eq. 1, one gets motion equations of the rotating elements and natural boundary conditions as follows:

$$
\begin{aligned}
& \rho A\left[-\ddot{x}+\ddot{\varphi} e_{v} \cos (\Omega t+\varphi+\beta)+\ddot{\varphi} e_{u} \sin (\Omega t+\varphi+\beta)\right. \\
& \left.-(\Omega+\dot{\varphi})^{2} e_{v} \sin (\Omega t+\varphi+\beta)+(\Omega+\dot{\varphi})^{2} e_{u} \cos (\Omega t+\varphi+\beta)\right] \\
& +k_{s} G A\left(x^{\prime \prime}-\theta_{y}^{\prime}\right)-F_{a} x^{\prime \prime}=0
\end{aligned}
$$

$$
\begin{aligned}
& \rho A\left[-\ddot{y}-\ddot{\varphi} e_{u} \cos (\Omega t+\varphi+\beta)+\ddot{\varphi} e_{v} \sin (\Omega t+\varphi+\beta)\right. \\
& \left.+(\Omega+\dot{\varphi})^{2} e_{u} \sin (\Omega t+\varphi+\beta)+(\Omega+\dot{\varphi})^{2} e_{v} \cos (\Omega t+\varphi+\beta)\right] \\
& +k_{s} G A\left(y^{\prime \prime}+\theta_{x}^{\prime}\right)-F_{a} y^{\prime \prime}=0
\end{aligned}
$$

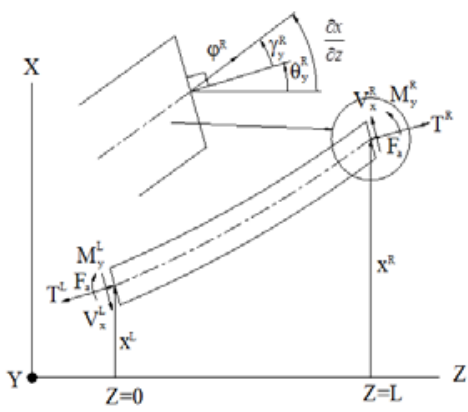

(a)

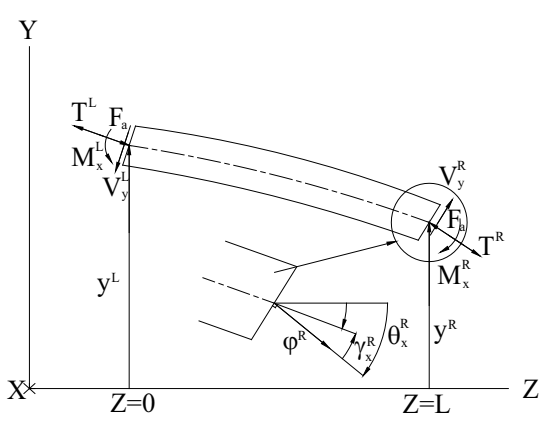

(b)

Figure 5. Forces, moments, and torques applying on a rotating element

$$
\begin{aligned}
& \rho I \ddot{\theta}_{x}+\frac{1}{2} \rho I_{p} \ddot{\varphi} \theta_{y}+\rho I_{p}(\Omega+\dot{\varphi}) \dot{\theta}_{y}-E I \theta_{x}^{\prime \prime}+k_{s} G A\left(\theta_{x}+y^{\prime}\right) \\
& +\rho \Delta\left[\ddot{\theta}_{y} \sin 2(\Omega t+\varphi+\beta)+2 \dot{\theta}_{y}(\Omega+\dot{\varphi}) \cos 2(\Omega t+\varphi+\beta)\right. \\
& \left.+\ddot{\theta}_{x} \cos 2(\Omega t+\varphi+\beta)-2 \dot{\theta}_{x}(\Omega+\dot{\varphi}) \sin 2(\Omega t+\varphi+\beta)\right] \\
& +E \Delta\left[-\theta_{y}^{\prime \prime} \sin 2(\Omega t+\varphi+\beta)-2 \theta_{y}^{\prime} \varphi^{\prime} \cos 2(\Omega t+\varphi+\beta)\right. \\
& \left.-\theta_{x}^{\prime \prime} \cos 2(\Omega t+\varphi+\beta)+2 \theta_{x}^{\prime} \varphi^{\prime} \sin 2(\Omega t+\varphi+\beta)\right]=0 \\
& \rho I \ddot{\theta}_{y}-\frac{1}{2} \rho I_{p} \ddot{\varphi} \theta_{x}-\rho I_{p}(\Omega+\dot{\varphi}) \dot{\theta}_{x}-E I \theta_{y}^{\prime \prime}+k_{s} G A\left(\theta_{y}-x^{\prime}\right) \\
& +\rho \Delta\left[\ddot{\theta}_{x} \sin 2(\Omega t+\varphi+\beta)+2 \dot{\theta}_{x}(\Omega+\dot{\varphi}) \cos 2(\Omega t+\varphi+\beta)\right. \\
& \left.-\ddot{\theta}_{y} \cos 2(\Omega t+\varphi+\beta)+2 \dot{\theta}_{y}(\Omega+\dot{\varphi}) \sin 2(\Omega t+\varphi+\beta)\right] \\
& +E \Delta\left[-\theta_{x}^{\prime \prime} \sin 2(\Omega t+\varphi+\beta)-2 \theta_{x}^{\prime} \varphi^{\prime} \cos 2(\Omega t+\varphi+\beta)\right. \\
& \left.+\theta_{y}^{\prime \prime} \cos 2(\Omega t+\varphi+\beta)-2 \theta_{y}^{\prime} \varphi^{\prime} \sin 2(\Omega t+\varphi+\beta)\right]=0 \\
& \rho I_{p} \ddot{\varphi}+\frac{1}{2} \rho I_{p} \ddot{\theta}_{x} \theta_{y}-\frac{1}{2} \rho I_{p} \ddot{\theta}_{y} \theta_{x}+\rho A\left[-\ddot{x} e_{v} \cos (\Omega t+\varphi+\beta)\right. \\
& -\ddot{x} e_{u} \sin (\Omega t+\varphi+\beta)+\ddot{y} e_{u} \cos (\Omega t+\varphi+\beta) \\
& \left.-\ddot{y} e_{v} \sin (\Omega t+\varphi+\beta)+e^{2} \ddot{\varphi}\right]-G I_{p} \varphi^{\prime \prime} \\
& +\rho \Delta\left[-2 \dot{\theta}_{x} \dot{\theta}_{y} \cos 2(\Omega t+\varphi+\beta)+\left(\dot{\theta}_{x}\right)^{2} \sin 2(\Omega t+\varphi+\beta)\right. \\
& \left.-\left(\dot{\theta}_{y}\right)^{2} \sin 2(\Omega t+\varphi+\beta)\right]+E \Delta\left[2 \theta_{x}^{\prime} \theta_{y}^{\prime} \cos 2(\Omega t+\varphi+\beta)\right. \\
& \left.-\left(\theta_{x}^{\prime}\right)^{2} \sin 2(\Omega t+\varphi+\beta)+\left(\theta_{y}^{\prime}\right)^{2} \sin 2(\Omega t+\varphi+\beta)\right]=0
\end{aligned}
$$

Note that the $\varphi$ and $x$ appeared in Eqs. 12, 15, and 16, so as $\varphi$ and $y$ appeared in Eqs. 13, 14, and 16. So there are the coupling effect between torsional displacement $\varphi$ 
and lateral displacements $(x, y)$. $(\Omega+\dot{\varphi}) \dot{\theta}_{y}$, and $\dot{\theta}_{x}(\Omega+\dot{\varphi})$ in Eqs. 14 , and 15, respectively present the gyroscopic effects.

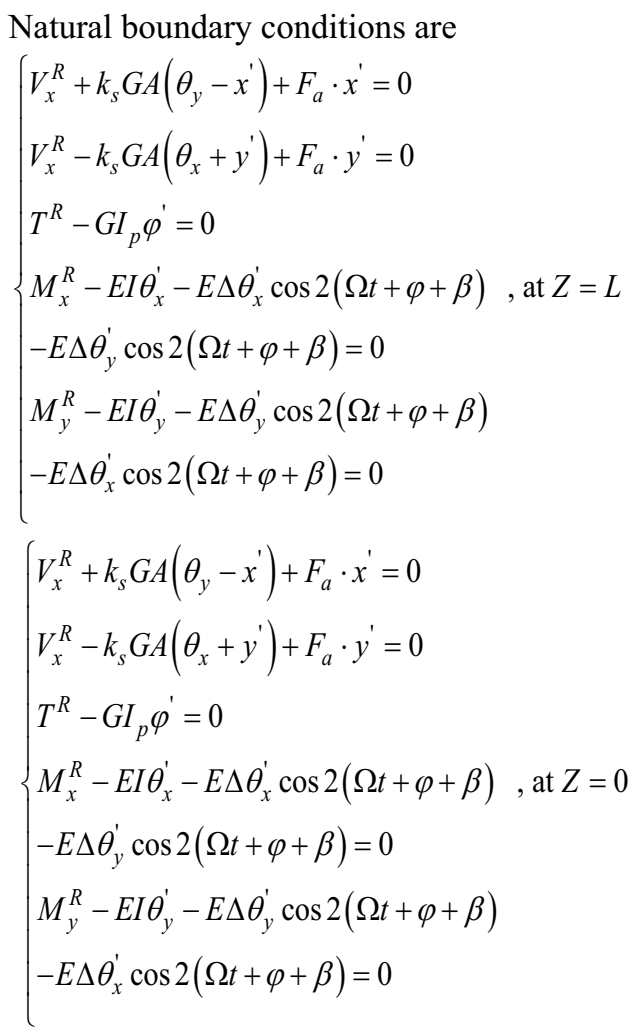

\section{Transfer Matrix of the Micro Drilling System}

The most important work of the transfer matrix method is to calculate the overall transfer matrix of the system. Based on it, the state vector including displacements, shear forces, moments, and torques at any position of the system can be evaluated. It is the multiplication of element transfer matrices that are developed by solving their motion equations.

The transfer matrix of pre-twisted rotating Timoshenko beam element is obtained by solving Eqs. 12-16. The solution process is presented below. Assuming the steady-state solutions of Eqs. 12-16 can be expressed in series form as

$$
\left\{\begin{array}{l}
x(Z, t)=x_{0}(Z)+\sum_{i=1}^{n} x_{i c}(Z) \cos i \Omega t+x_{i s}(Z) \sin i \Omega t \\
y(Z, t)=y_{0}(Z)+\sum_{i=1}^{n} y_{i c}(Z) \cos i \Omega t+y_{i s}(Z) \sin i \Omega t \\
\theta_{x}(Z, t)=\theta_{x, 0}(Z)+\sum_{i=1}^{n} \theta_{x, i c}(Z) \cos i \Omega t+\theta_{x, i s}(Z) \sin i \Omega t \\
\theta_{y}(Z, t)=\theta_{y, 0}(Z)+\sum_{i=1}^{n} \theta_{y, i c}(Z) \cos i \Omega t+\theta_{y, i s}(Z) \sin i \Omega t \\
\varphi(Z, t)=\varphi_{0}(Z)+\sum_{i=1}^{n} \varphi_{i c}(Z) \cos i \Omega t+\varphi_{i s}(Z) \sin i \Omega t
\end{array}\right.
$$

where,

$$
\begin{aligned}
& x_{0}(Z), y_{0}(Z), \theta_{x, 0}(Z), \theta_{y, 0}(Z), \varphi_{0}(Z), x_{i c}(Z), x_{i s}(Z), \\
& y_{i c}(Z), y_{i s}(Z), \theta_{x, i c}(Z), \theta_{x, i s}(Z), \theta_{y, i c}(Z), \theta_{y, i s}(Z), \varphi_{i c}(Z),
\end{aligned}
$$
and $\varphi_{i s}(Z)$ are the mode functions of the relative $0^{\text {th }}$ order and $n \times$ harmonic whirl with respect to the static coordinate system of the Timoshenko beam element.

$$
\begin{aligned}
\mathbf{X}(\mathbf{Z})= & {\left[x_{0}(Z) x_{1 c}(Z) \cdots x_{n c}(Z) x_{1 s}(Z) \cdot x_{n s}(Z)\right.} \\
& y_{0}(Z) y_{1 c}(Z) \cdot y_{n c}(Z) y_{1 s}(Z) \cdot y_{n s}(Z) \\
& \theta_{x, 0}(Z) \theta_{x, 1 c}(Z) \cdot \theta_{x, n c}(Z) \theta_{x, 1 s}(Z) \cdot \theta_{x, n s}(Z) \\
& \theta_{y, 0}(Z) \theta_{y, 1 c}(Z) \cdot \theta_{y, n c}(Z) \theta_{y, 1 s}(Z) \cdots \theta_{y, n s}(Z) \\
& \left.\varphi_{0}(Z) \varphi_{1 c}(Z) \cdots \varphi_{n c}(Z) \varphi_{1 s}(Z) \cdots \varphi_{n s}(Z)\right]^{T}
\end{aligned}
$$

The size of $\mathbf{X}(\mathbf{Z})$ is $(10 n+5) \times 1$. Replacing Eq. 19 into Eqs. 12-16, ignoring nonlinear terms, and equating the same harmonic term, the following matrix equation can be obtained.

$$
\mathbf{E}_{\mathbf{2}} \mathbf{X}^{\prime \prime}(\mathbf{Z})+\mathbf{E}_{\mathbf{1}} \mathbf{X}^{\prime}(\mathbf{Z})+\mathbf{E}_{\mathbf{0}} \mathbf{X}(\mathbf{Z})=\mathbf{F}_{\mathbf{0}}
$$

where, $\mathbf{X}^{\prime \prime}(\mathbf{Z}), \mathbf{X}^{\prime}(\mathbf{Z})$ are second and first order derivatives of $\mathbf{X}(\mathbf{Z})$ with respect to $Z$ coordinate, respectively. $\mathbf{E}_{\mathbf{2}}, \mathbf{E}_{\mathbf{1}}$, and $\mathbf{E}_{\mathbf{0}}$ are matrices with size of $(10 n+5) \times(10 n+5)$. The size of matrix $\mathbf{F}_{\mathbf{0}}$ is $(10 n+5) \times 1$. The general solutions of Eq .21 are the sum of homogeneous and particular solutions.

$$
\mathbf{X}(\mathbf{Z})=\mathbf{X}(\mathbf{Z})^{h}+\mathbf{X}(\mathbf{Z})^{p}
$$

The homogeneous solution $\mathbf{X}(\mathbf{Z})^{h}$ is [11]

$$
\mathbf{X}(\mathbf{Z})^{h}=\sum_{i=1}^{k} C_{i} \mathbf{X}_{i}^{h} e^{\lambda_{i} Z}
$$

where, $\lambda_{i}$ is eigen-value, $\mathbf{X}_{i}^{h}$ is eigen-vector corresponding to $\lambda_{i}$, which are solutions of Eq. $24 ; C_{i}$ is an undetermined constant.

$$
\left\{\lambda\left[\begin{array}{cc}
\mathbf{0} & \mathbf{E}_{\mathbf{2}} \\
\mathbf{E}_{\mathbf{2}} & \mathbf{E}_{\mathbf{1}}
\end{array}\right]_{k \times k}-\left[\begin{array}{cc}
\mathbf{E}_{\mathbf{2}} & \mathbf{0} \\
\mathbf{0} & \mathbf{E}_{\mathbf{0}}
\end{array}\right]_{k \times k}\right\}\left[\begin{array}{l}
\lambda \mathbf{X}^{h} \\
\mathbf{X}^{h}
\end{array}\right]_{k \times 1}=\left[\begin{array}{l}
\mathbf{0} \\
\mathbf{0}
\end{array}\right]_{k \times 1}
$$

where, $k=20 n+10$.

The particular solution is

$$
\begin{aligned}
\mathbf{X}(\mathbf{Z})^{h}= & {\left[\begin{array}{llllllll}
x_{0}^{p} & x_{1 c}^{p} \cdot x_{n c}^{p} & x_{1 s}^{p} \cdot \cdot x_{n s}^{p} & y_{0}^{p} & y_{1 c}^{p} \cdot \cdot y_{n c}^{p} & y_{1 s}^{p} \cdot \cdot y_{n s}^{p} \\
& \theta_{x, 0}^{p} & \theta_{x, 1 c}^{p} \cdot \theta_{x, n c}^{p} & \theta_{x, 1 s}^{p} \cdot \cdots & \theta_{x, n s}^{p} & \theta_{y, 0}^{p} & \theta_{y, 1 c}^{p} \cdot \theta_{y, n c}^{p} \\
& \theta_{y, 1 s}^{p} \cdot \cdots & \theta_{y, n s}^{p} & \varphi_{0}^{p} & \varphi_{1 c}^{p} \cdot \cdot \varphi_{n c}^{p} & \varphi_{1 s}^{p} \cdot \cdot \varphi_{n s}^{p}
\end{array}\right] }
\end{aligned}
$$


where

$$
\begin{aligned}
& x_{1 s}^{p}=-y_{1 c}^{p}=e_{v} \cos \beta+e_{u} \sin \beta, x_{1 c}^{p}=y_{1 s}^{p}=e_{v} \sin \beta-e_{u} \cos \beta, \\
& y_{2 c}^{p}=x_{2 s}^{p}=-\frac{g \Delta \cos 2 \beta}{4 \Omega^{2} I}, y_{2 s}^{p}=x_{2 c}^{p}=-\frac{\Delta g \sin 2 \beta}{4 \Omega^{2} I}, \\
& \theta_{x, 2 c}^{p}=\theta_{y, 2 s}^{p}=\frac{\Delta \rho g \cos 2 \beta}{k_{s} G I} Z, \theta_{x, 2 s}^{p}=-\theta_{y, 2 c}^{p}=\frac{\Delta \rho g \sin 2 \beta}{k_{s} G I} Z,
\end{aligned}
$$

other elements $=0$.

Substituting Eqs. 25, and 23 into Eq. 22, one gets

$$
\mathbf{X}(\mathbf{Z})=[G(Z)]\left[\begin{array}{l}
\mathbf{C} \\
1
\end{array}\right]
$$

where $[G(Z)]$ is the matrix function of $Z$ with the size of $\quad(10 n+5) \times(20 n+11), \quad \mathbf{C}=\left[\begin{array}{llll}C_{1} & C_{2} & \cdots & C_{20 n+10}\end{array}\right]^{T}$, where, $C_{i}$ the undetermined constant in Eq. 23. Hence the displacement state vectors at two ends of the drill flute elements are $\quad \mathbf{X}^{R}=\mathbf{X}(Z=L)=\left[G_{L}\right]\left[\begin{array}{l}\mathbf{C} \\ 1\end{array}\right]$

$$
\mathbf{X}^{L}=\mathbf{X}(Z=0)=\left[G_{0}\right]\left[\begin{array}{l}
\mathbf{C} \\
1
\end{array}\right]
$$

where $\left[G_{L}\right]=[G(Z=L)],\left[G_{0}\right]=[G(Z=0)]$.

Assuming the solutions of Eqs. 17, and 18 are described in Fourier series form as

$$
\left\{\begin{array}{l}
V_{x}(Z, t)=V_{x, 0}(Z)+\sum_{i=1}^{n} V_{x, i c}(Z) \cos i \Omega t+V_{x, i s}(Z) \sin i \Omega t \\
V_{y}(Z, t)=V_{y, 0}(Z)+\sum_{i=1}^{n} V_{y, i c}(Z) \cos i \Omega t+V_{y, i s}(Z) \sin i \Omega t \\
M_{x}(Z, t)=M_{x, 0}(Z)+\sum_{i=1}^{n} M_{x, i c}(Z) \cos i \Omega t+M_{x, i s}(Z) \sin i \Omega t \\
M_{y}(Z, t)=M_{y, 0}(Z)+\sum_{i=1}^{n} V_{y, i c}(Z) \cos i \Omega t+M_{y, i s}(Z) \sin i \Omega t \\
T(Z, t)=T_{0}(Z)+\sum_{i=1}^{n} T_{i c}(Z) \cos i \Omega t+T_{i s}(Z) \sin i \Omega t
\end{array}\right.
$$

The mode function vector of the general force $\mathbf{F}(Z)$ is defined as below

$$
\begin{aligned}
\mathbf{F}(Z)= & {\left[V_{x, 0}(Z) V_{x, 1 c}(Z) \cdot V_{x, n c}(Z) V_{x, 1 s}(Z) \cdot V_{x, n s}(Z)\right.} \\
& V_{y, 0}(Z) V_{y, 1 c}(Z) \cdot V_{y, n c}(Z) V_{y, 1 s}(Z) \cdot V_{y, n s}(Z) \\
& \mathrm{M}_{x, 0}(Z) M_{x, 1 c}(Z) \cdot M_{x, n c}(Z) M_{x, 1 s}(Z) \cdot M_{x, n s}(Z) \\
& M_{y, 0}(Z) M_{y, 1 c}(Z) \cdot M_{y, n c}(Z) M_{y, 1 s}(Z) \cdot M_{y, n s}(Z) \\
& \left.T_{0}(Z) T_{1 c}(Z) \cdot T_{n c}(Z) T_{1 c}(Z) \cdot T_{n c}(Z)\right]
\end{aligned}
$$

Substituting Eqs. 19, 27, 29, and 30 into Eq. 17, and equating the coefficients of the same harmonic term, one obtained the vector of general force general force $\mathbf{F}(Z)$ at the right end of the Timoshenko beam element.

$$
\mathbf{F}^{R}=\left[H_{L}\right]\left[\begin{array}{l}
\mathbf{C} \\
1
\end{array}\right]
$$

Similarly, Substituting Eqs. 19, 28, 29, and 30 into Eq. 18, the vector $\mathbf{F}(Z)$ at the left end is

$$
\mathbf{F}^{L}=\left[H_{0}\right]\left[\begin{array}{l}
\mathbf{C} \\
1
\end{array}\right]
$$

where $\left[H_{L}\right],\left[H_{0}\right]$ are matrices with the size of $(10 n+5) \times(20 n+11)$.

Combining Eqs. 27, and 31, one gets

$$
\left[\begin{array}{l}
\mathbf{X}^{R} \\
\mathbf{F}^{R} \\
1
\end{array}\right]=\left[\begin{array}{l}
G_{L} \\
H_{L} \\
M_{U}
\end{array}\right]\left[\begin{array}{l}
\mathbf{C} \\
1
\end{array}\right]
$$

where

$$
M_{U}=\left[\begin{array}{lll}
0 & 0 & \cdots
\end{array}\right]_{1 \times(20 n+11)}
$$

Let $\left[\mathbf{S}^{R}\right]=\left[\begin{array}{c}\mathbf{X}^{R} \\ \mathbf{F}^{R}\end{array}\right],\left[M_{L}\right]=\left[\begin{array}{c}G_{L} \\ H_{L} \\ M_{U}\end{array}\right]$, and inserting them into Eq. 33 yields

$$
\left[\begin{array}{l}
\mathbf{S}^{R} \\
1
\end{array}\right]=\left[M_{L}\right]\left[\begin{array}{l}
\mathbf{C} \\
1
\end{array}\right]
$$

Similarly, combining Eqs 28, and 32, one gets

$$
\left[\begin{array}{l}
\mathbf{S}^{L} \\
1
\end{array}\right]=\left[M_{0}\right]\left[\begin{array}{l}
\mathbf{C} \\
1
\end{array}\right]
$$

where, $\left[M_{L}\right],\left[M_{0}\right]$ are matrices with the size of $(20 n+11) \times(20 n+11)$. From Eqs. 34, and 35, it yields

$$
\left[\begin{array}{l}
\mathbf{S}^{R} \\
1
\end{array}\right]=\left[M_{L}\right]\left[\begin{array}{l}
\mathbf{C} \\
1
\end{array}\right]=\left[M_{L}\right]\left[M_{0}\right]^{-1}\left[\begin{array}{l}
\mathbf{S}^{L} \\
1
\end{array}\right]=\left[T^{s}\right]\left[\begin{array}{l}
\mathbf{S}^{L} \\
1
\end{array}\right]
$$

where $\left[M_{0}\right]^{-1}$ is the inverse matrix of matrix $\left[M_{0}\right]$, $\left[T^{s}\right]=\left[M_{L}\right]\left[M_{0}\right]^{-1}$ is called as transfer matrix of the pre-twisted rotating Timoshenko beam element. From it, the relation of the state vector $\mathbf{S}$ including displacements, shear forces, moments, and torques at two element ends is developed. So far, transfer matrices of spindle and micro drill elements are derived from the transfer matrix of the of the pre-twisted rotating Timoshenko beam element. Transfer matrix of the bearing will be considered next.

The bearings supporting the micro drilling system are considered to be linear and isotropic. The bearing as shown in Fig. 6 creates forces $F_{x}^{b}, F_{y}^{b}$, bending moments 
$M_{x}^{b}, M_{y}^{b}$, and torque $T^{b}$ applying on the spindle. They are calculated by the following Eq. 37.

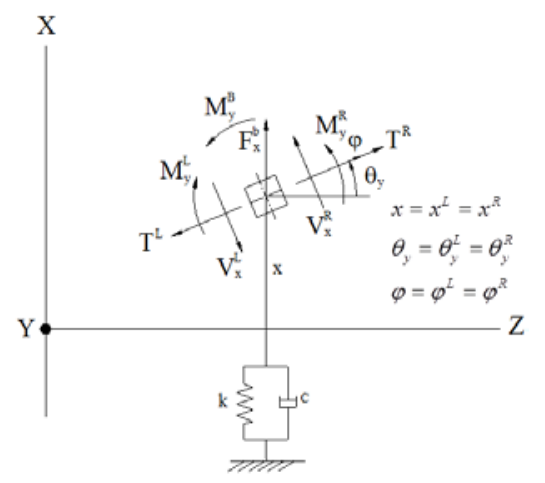

(a)

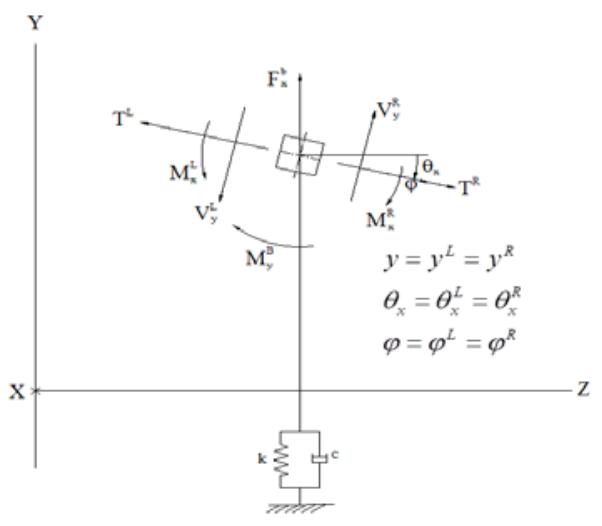

(b)

Figure 6. Forces, moments, and torques applying on a bearing node

$$
\left\{\begin{array}{l}
{\left[\begin{array}{l}
F_{x}^{b} \\
F_{y}^{b}
\end{array}\right]=-\left[\begin{array}{ll}
K_{x x} & K_{x y} \\
K_{y x} & K_{y y}
\end{array}\right]\left[\begin{array}{l}
x \\
y
\end{array}\right]-\left[\begin{array}{ll}
C_{x x} & C_{x y} \\
C_{y x} & C_{y y}
\end{array}\right]\left[\begin{array}{c}
\dot{x} \\
\dot{y}
\end{array}\right]} \\
{\left[\begin{array}{l}
M_{x}^{b} \\
M_{y}^{b}
\end{array}\right]=-\left[\begin{array}{ll}
K_{\theta x x} & K_{\theta x y} \\
K_{\theta y x} & K_{\theta y y}
\end{array}\right]\left[\begin{array}{l}
\theta_{x} \\
\theta_{y}
\end{array}\right]-\left[\begin{array}{ll}
C_{\theta x x} & C_{\theta x y} \\
C_{\theta y x} & C_{\theta y y}
\end{array}\right]\left[\begin{array}{c}
\dot{\theta}_{x} \\
\dot{\theta}_{y}
\end{array}\right]} \\
T^{b}=-K_{\varphi} \varphi-C_{\varphi} \dot{\varphi}
\end{array}\right.
$$

The equilibrium equations of the force, bending moment and torque acting on the bearing node as shown in Fig. 6 are

$$
\left\{\begin{aligned}
{\left[\begin{array}{l}
V_{x}^{R} \\
V_{y}^{R}
\end{array}\right] } & =\left[\begin{array}{l}
V_{x}^{L} \\
V_{y}^{L}
\end{array}\right]-\left[\begin{array}{l}
F_{x}^{b} \\
F_{y}^{b}
\end{array}\right] \\
& =\left[\begin{array}{l}
V_{x}^{L} \\
V_{y}^{L}
\end{array}\right]+\left[\begin{array}{ll}
K_{x x} & K_{x y} \\
K_{y x} & K_{y y}
\end{array}\right]\left[\begin{array}{l}
x \\
y
\end{array}\right]+\left[\begin{array}{ll}
C_{x x} & C_{x y} \\
C_{y x} & C_{y y}
\end{array}\right]\left[\begin{array}{c}
\dot{x} \\
\dot{y}
\end{array}\right] \\
{\left[\begin{array}{l}
M_{x}^{R} \\
M_{y}^{R}
\end{array}\right] } & =\left[\begin{array}{l}
M_{x}^{L} \\
M_{y}^{L}
\end{array}\right]-\left[\begin{array}{l}
M_{x}^{b} \\
M_{y}^{b}
\end{array}\right] \\
& =\left[\begin{array}{l}
M_{x}^{L} \\
M_{y}^{L}
\end{array}\right]+\left[\begin{array}{ll}
K_{\theta x x} & K_{\theta x y} \\
K_{\theta y x} & K_{\theta y y}
\end{array}\right]\left[\begin{array}{l}
\theta_{x} \\
\theta_{y}
\end{array}\right]+\left[\begin{array}{ll}
C_{\theta x x} & C_{\theta x y} \\
C_{\theta y x} & C_{\theta y y}
\end{array}\right]\left[\begin{array}{l}
\dot{\theta}_{x} \\
\dot{\theta}_{y}
\end{array}\right] \\
T^{R}= & T^{L}-T^{b}=T^{L}+K_{\varphi} \varphi+C_{\varphi} \dot{\varphi}
\end{aligned}\right.
$$

Replacing Eqs. 19, and 29 into Eq. 38, and equating the coefficients of the same harmonic term, one obtained

$$
\left[\begin{array}{l}
\mathbf{S}^{R} \\
1
\end{array}\right]=\left[T^{b}\right]_{(20 n+11) \times(20 n+11)}\left[\begin{array}{l}
\mathbf{S}^{L} \\
1
\end{array}\right]
$$

where the state vector $\mathbf{S}$ contains all coefficients of Eqs. 19, and 29. The $\left[T^{b}\right]$ is the transfer matrix of the bearing. Then the overall transfer matrix of the whole system is the multiplication of the transfer matrices of all elements from the tip of the micro drill to its end.

According to the modified transfer matrix method, the state vectors at two ends of the micro drilling system are described as the following.

$$
\left[\begin{array}{l}
\mathbf{X}^{R} \\
\mathbf{F}^{R} \\
1
\end{array}\right]=[U]\left[\begin{array}{l}
\mathbf{X}^{L} \\
\mathbf{F}^{L} \\
1
\end{array}\right]
$$

where $[U]$ is the total transfer matrix of the system, and it is the multiplication of transfer matrices of elements. From Fig. 1, and Table 1, one gets

$$
[U]=[T]_{A}[T]_{B}[T]_{C}[T]_{D}[T]_{S_{1}}[T]_{C_{1}}[T]_{S_{2}}[T]_{C_{2}}[T]_{F_{1}}
$$

where $\quad[T]_{A},[T]_{B},[T]_{C},[T]_{D},[T]_{S_{1}},[T]_{C_{1}},[T]_{S_{2}},[T]_{C_{2}}$, and $[T]_{F_{1}}$ are the transfer matrices of parts A, B, C, D, S $\mathrm{C}_{1}, \mathrm{~S}_{2}, \mathrm{C}_{2}$, and $\mathrm{F}_{1}$, respectively.

The micro drill flute $F_{1}$ shown in Fig 7(a) is divided into small elements, so its transfer matrix $[T]_{F_{1}}$ is the multiplication of transfer matrices of these elements. The transfer matrix of each flute element is derived from the transfer matrix of the of the pre-twisted rotating Timoshenko beam element. To calculate it, it is necessary to evaluate the area moments of inertia $\left(I_{u}, I_{v}\right)$ about its principal axes of the flute cross-section as well as its polar moments of inertia $I_{p}$, and the twist angle $\beta$ between $U$ principal axis and $x$-axis. Because the drill flute is formed by sweeping helically a flute cross-section, 
every drill flute element has the same area moments of inertia. Therefore, to be simple, $I_{u}, I_{v}$, and $I_{p}$ are calculated at the flute cross-section perpendicular to the drill axis at the drill tip $\mathrm{O}$ as shown in Fig 7. Developing the profile of the cross section in Fig 7(a) is referred to the reference $[16,17]$. Its moments of inertia $I_{u}, I_{v}$, and $I_{p} \quad$ are evaluated by Solidworks software, and its principal axes $U, V$ is coincident to the axes $x$ and $y$ of the fixed coordinate system, respectively [18]. The twist angle $\beta$ of the cross section of the flute element with coordinate $Z_{i}$ as shown in Fig 7(b) is calculated by the following equation: $\beta=Z_{i} \frac{\tan \left(h_{0}\right)}{R}$, where $R, h_{0}$ are radius, helical angle of the micro drill, respectively.

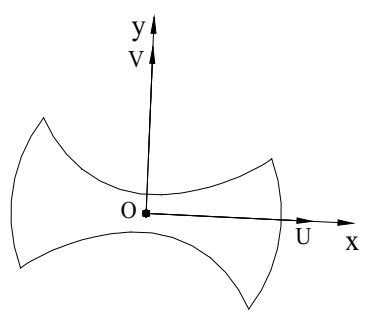

(a) Flute elements

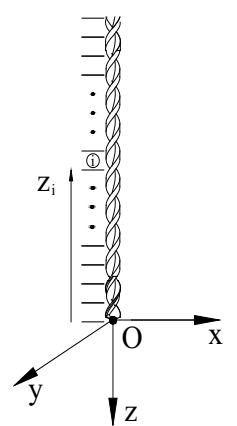

(b) Flute cross- section
Figure 7. Micro drill flute

To be simple, the conical part of the micro drill is considered as the sum of small cylinders as shown in Fig. 8. Hence, the transfer matrix of the conical part is the multiplication of transfer matrices of small cylinders. The area principal moments of inertia $\left(I_{u}, I_{v}\right)$, area polar moment of inertia $I_{p}$, area $A$, and initial twist angle $\beta$ of a cylinder are calculated as follows: $I=I_{u}=I_{v}=\frac{\pi r^{4}}{4}, I_{p}=\frac{\pi r^{4}}{2}, A=\pi r^{2}, \beta=0$, where $r$ is the radius of the cross section.

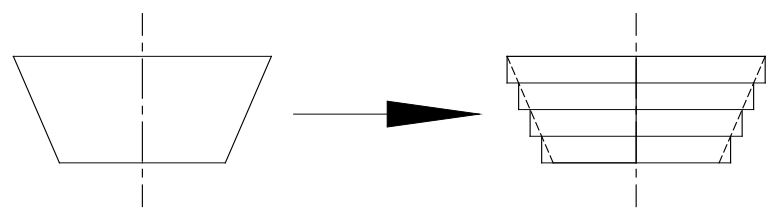

Figure 8. Approximation of conical part

The transfer matrix of spindle part A including two bearings, and three cylinder portions is

$$
[T]_{A}=[T]_{L 3}[T]^{B}[T]_{A 2}[T]^{B}[T]_{L 3}
$$

\section{Dynamic analysis of the micro drilling system}

The vibration at the micro drill tip has the most important impact to the quality of drilled holes, and the cutting life time of the micro drill. Therefore, the dynamic analysis is carried out at the drill tip in this research. Namely, the state vectors containing displacements $\left(x, \mathrm{y}, \theta_{x}, \theta_{y}, \varphi\right)$, and the critical speeds of the system are evaluated.

After calculating all transfer matrices of system elements, the overall transfer matrix $[U]$ is calculated. Eq. 40 can be rewritten as Eq. 43

$$
\left[\begin{array}{l}
\mathbf{X}^{R} \\
\mathbf{F}^{R} \\
1
\end{array}\right]=\left[\begin{array}{ccc}
U_{11} & U_{12} & \mathbf{u}_{\mathbf{1}} \\
U_{21} & U_{22} & \mathbf{u}_{\mathbf{2}} \\
\mathbf{0} & \mathbf{0} & 1
\end{array}\right]\left[\begin{array}{l}
\mathbf{X}^{L} \\
\mathbf{F}^{L} \\
1
\end{array}\right]
$$

where $U_{11}, U_{12}, U_{21}, U_{22}$ are matrices with the size of $(10 n+5) \times(10 n+5) . \mathbf{u}_{1}, \mathbf{u}_{2}$ are vectors with the size of $(10 n+5) \times 1$. The $\mathbf{0}$ is zero vector with the size of $1 \times(10 n+5)$. Because there is no external force applying on the left end of the drilling system, one obtains $\mathbf{F}^{L}=\left[\begin{array}{llll}0 & 0 & \cdots & 0\end{array}\right]_{(10 n+5) \times 1}$. Only torque $T$ is the external force applying on the right end or drill tip of the system,

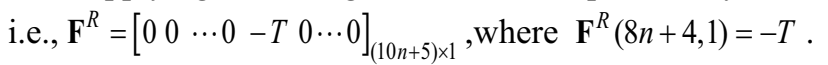
From Eq. 43, one obtains

$$
\begin{aligned}
\mathbf{F}^{R}= & U_{21} \mathbf{X}^{L}+U_{21} \mathbf{F}^{L}+\mathbf{u}_{2}=U_{21} \mathbf{X}^{L}+\mathbf{u}_{2} \\
& \Rightarrow \mathbf{X}^{L}=U_{21}^{-1}\left(\mathbf{F}^{R}-\mathbf{u}_{2}\right) \\
\mathbf{X}^{R} & =U_{11} \mathbf{X}^{L}+U_{12} \mathbf{F}^{L}+\mathbf{u}_{1}=U_{11} \mathbf{X}^{L}+\mathbf{u}_{1}
\end{aligned}
$$

The vector $\mathbf{X}^{R}$ containing the displacements $(x, \mathrm{y}$ $\left., \theta_{x}, \theta_{y}, \varphi\right)$ at the drill tip are calculated, i.e., the lateral and torsional displacements at the drill tip are evaluated.

Because lateral displacements including $x$ and $y$, and torsional displacement $\varphi$ play the most important role to the quality of the micro drill, they were analyzed in this section. The amplitude of the lateral (bending) displacement is calculated as below.

$$
\text { Lateral amplitude }=\text { maximum value of } \sqrt{x^{2}+y^{2}}
$$

where $x$ and $y$ are evaluated by Eq. 19 .

We chose order of whirling at the drill tip to be $n=2$. With the given parameters in Table 2, the lateral and torsional displacements are obtained. According to Fig. 9 $(\mathrm{a}, \mathrm{b})$, the lateral and torsional amplitudes is $5.39 \cdot 10^{-8} \mathrm{~m}$ and $7.4101769 \cdot 10^{-7} \mathrm{rad}$ under $30000 \mathrm{rpm}$, respectively. 
Table 2. Parameters of the micro drilling system

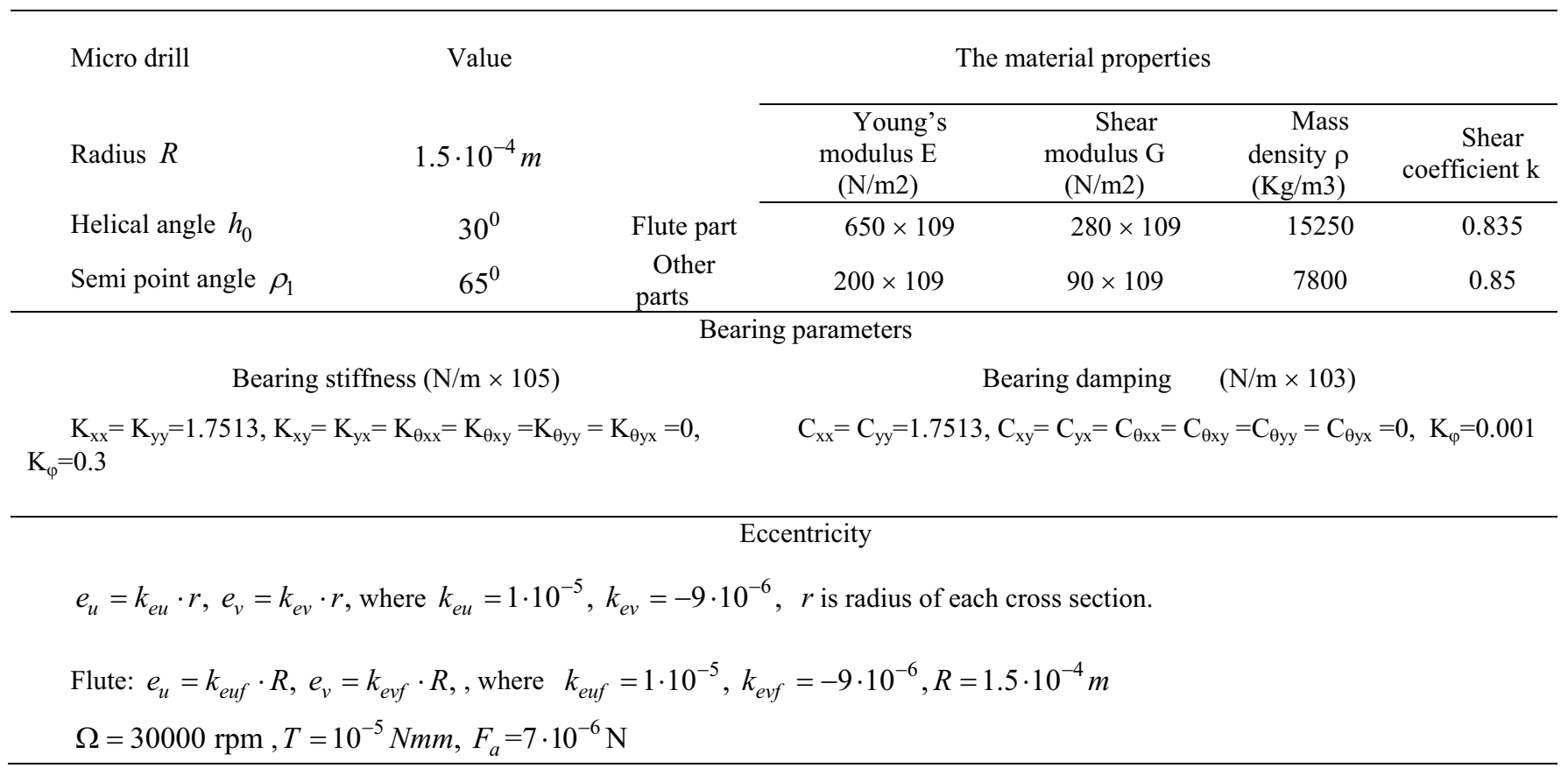

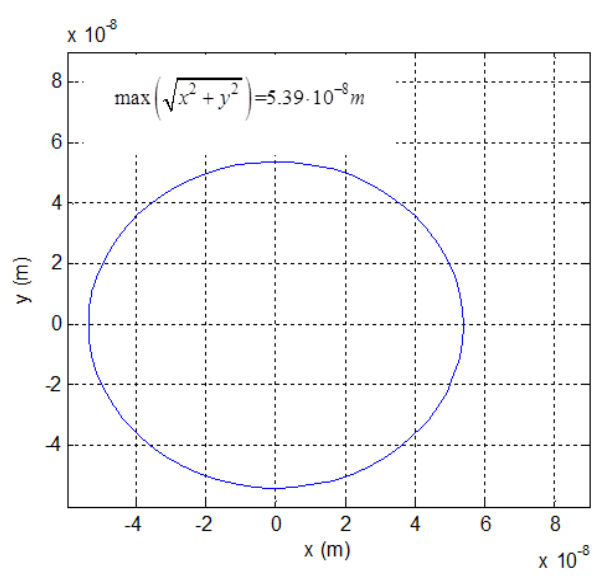

(a) Lateral displacement

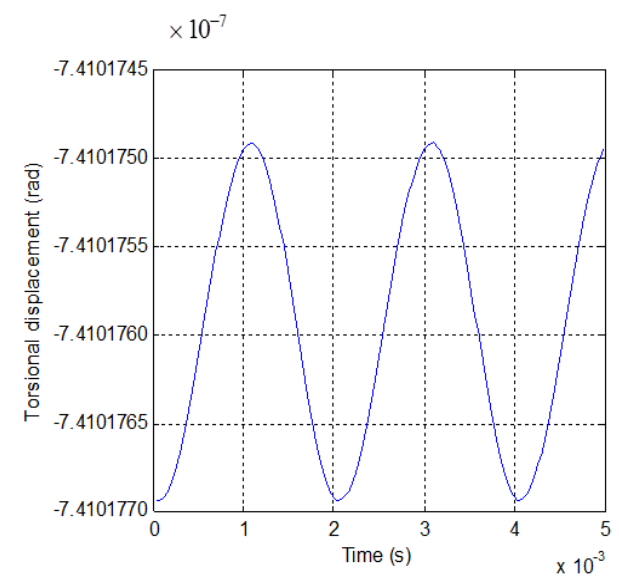

(b) Torsional displacement

Figure 9. Effects of external forces to displacements
Fig. 10 shows the lateral and torsional amplitudes. It shows that the lateral and torsional displacements are under resonance at rotating speed of $83080 \mathrm{rpm}$. Fig 11 shows the lateral and torsional displacement at the critical speed.

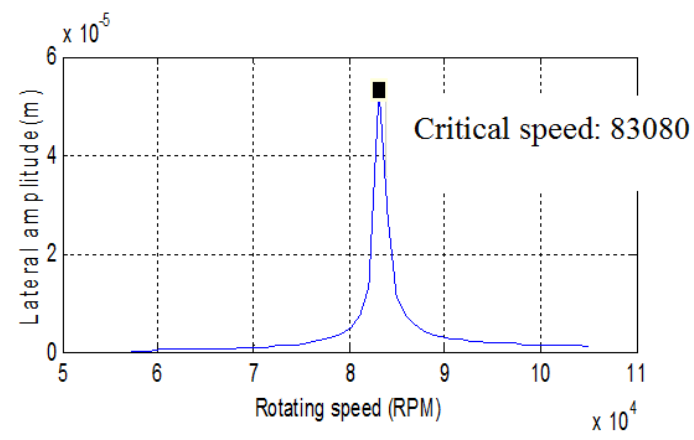

(a) Lateral amplitude

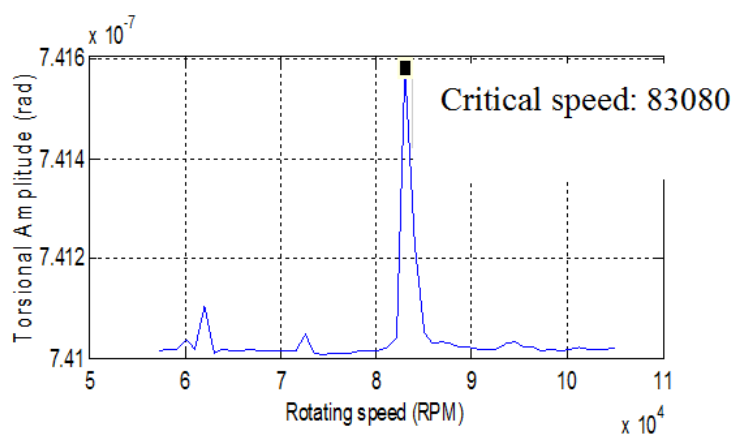

(b) Torsional amplitude

Figure 10. Effects of external forces to displacement amplitudes 


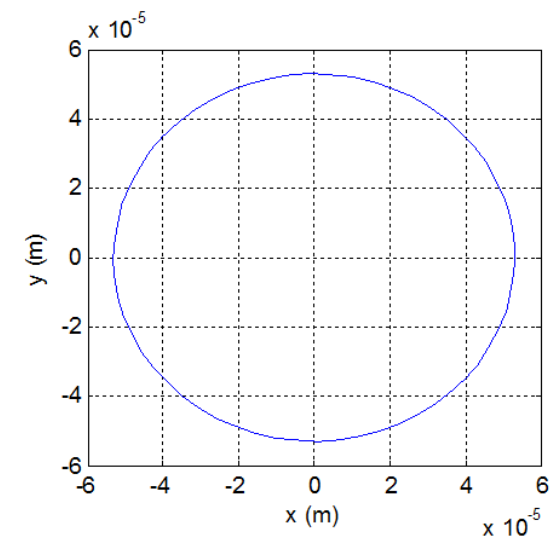

(a) Lateral displacement

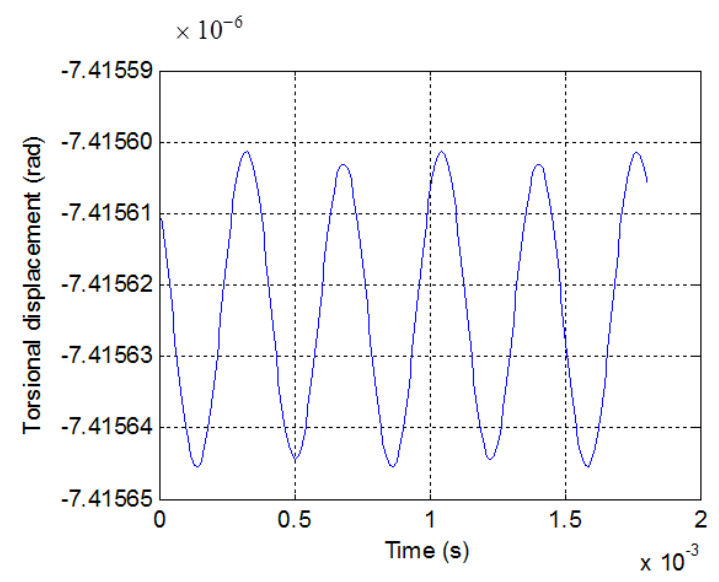

(b) Torsional displacement

Figure 11. Displacements at critical speed

\section{Conclusions}

The dynamic analysis of the micro drilling system was implemented by the new approach, modified transfer matrix, in this research. The micro drilling system is modeled as pre-twisted rotating Timoshenko beam elements using a continuous-system concept. Each element could include linear bearings, axial drilling force, gyroscopic moments, and eccentricity, especially coupling effect between lateral and torsional displacements. Therefore, the method has higher accuracy in evaluating the displacements and critical speeds of the system. The numerical example about effects of external forces, eccentricity, and bearings on lateral and torsional displacements and critical speeds of the system at the micro drill tip was presented in this research.

\section{References}

1. Y. Zhaojun, L. Wei, C. Yanhong, and W. Lijiang, Study for increasing micro-drill reliability by vibrating drilling, Reliab. Eng. Syst. Safe. 61, 5 (1998)
2. J. Chae, S. S. Park, and T. Freiheit, Investigation of micro-cutting operations, Int. J. Mach. Tool. Manu. 46, 20(2006)

3. T. Masuzawa, State of the Art of Micromachining, CIRP Annals - Manufacturing Technology, 49, 16(2000)

4. C. K. Yu, C. H. Chang, Y. P. Ping, Y. K. Tien, and H. B. Wun, Natural properties in a micro drill cutting into bones, Life Sci. J. 6, 6(2009)

5. B.-W. Huang, The Drilling Vibration Behavior of a Twisted Microdrill, J. Manuf. Sci. Eng. 126,8 (2005)

6. Z. Yang, Q. Tan, and L. Wang, Principle of precision micro-drilling with axial vibration of low frequency, Int. J. Prod. Res. 40, 7 (2002)

7. Y. Gong, K. F. Ehmann, and C. Lin, Analysis of dynamic characteristics of micro-drills, J. Mater. Process. Technol. 141, 9(2003)

8. P. Yongchen, T. Qingchang, and Y. Zhaojun, A study of dynamic stresses in micro-drills under highspeed machining, Int. J. Mach. Tool. Manu. 46,9 (2006)

9. S. Filiz and O. Burak Ozdoganlar, A model for bending, torsional, and axial vibrations of micro- and macro-drills including actual drill geometry-part I: model development and numerical solution, J. Manuf. Sci. Eng.132,(2010)

10. [10] S. Filiz and O. B. Ozdoganlar, A model for bending, torsional, and axial vibrations of micro- and macro-drills including actual drill geometry-part II: model validation and application, J. Manuf. Sci. Eng. 132,(2010)

11. S.-C. Hsieh, J.-H. Chen, and A.-C. Lee, A modified transfer matrix method for the coupling lateral and torsional vibrations of symmetric rotor-bearing systems, J. Sound Vib. 289, 10( 2006)

12. H. P. Lee, Dynamic response of a rotating timoshenko shaft subject to axial forces and moving loads, J. Sound Vib. 181, 9(1995)

13. D.-M. Ku, Finite element analysis of whirl speeds for rotor-bearing systems with internal damping, Mech. Syst. Sig. Process., 12, (1998)

14. Q. H. Qin and C. X. Mao, Coupled torsional-flexural vibration of shaft systems in mechanical engineering-I. Finite element model, Comput. Struct. 58, 9(1996)

15. Y. Kang, Y.-P. Shih, and A.-C. Lee, Investigation on the steady-state responses of asymmetric rotors, J. Sound Vib. 114, 7(1992)

16. C. Lin, S. Kang, and K. Ehmann, "Planar micro-drill point design and grinding methods," Trans. North Am. Manuf. Res. Inst. SME, 20, 7(1992)

17. S.-C. Hsieh, J.-H. Chen, and A.-C. Lee, A modified transfer matrix method for the coupled lateral and torsional vibrations of asymmetric rotor-bearing systems, J. Sound Vib. 312, 9(2008)

18. A.-C. Lee, D.-T. Nguyen, and G.-T. Wu, Analyses of a new four-facet drill, Int. J. Adv. Manuf. Technol.75,14(2014) 